\title{
DAS HYMENOPHORALE TRAMA BEI DEN AGARICALES
}

\author{
G. A. C. DOUWES UND J. A. VON ARX \\ (Botanisches Museum und Herbarium, Utrecht, und Centraalbureau voor \\ Schimmelcultures, Baarn)
}

(eingegangen am 2. Februar 1965)

\begin{abstract}
AbstraGt
Short descriptions and camera lucida drawings are given of cross-sections through the hymenophoral trama (lamellae or tubes) of 24 Basidiomycetes. These represent all the 16 Families of the Agaricales sensu Singer (1962). The typical members of the Agaricales in a limited definition have to be characterised by a more or less regular or bilateral trama with enlarged cells, or with swollen cellwalls in the Boletaceae, Strobilomycetaceae, Gomphidiaceae and Paxillaceae. In species of the genera Polyporus, Pleurotus (Polyporaceae), Lentinellus and Crepidotus such enlarged or swollen cells are lacking, the trama is irregular and is composed of a dense network of thickwalled hyphae. These genera and others like Schizophyllum better can be classified in the Aphyllophorales.
\end{abstract}

\section{EINLEITUNG}

In den letzten Jahren sind zwei umfangreiche Arbeiten über den Entwicklungsgang und die Systematik der zu den Agaricales zu stellenden Basidiomyceten erschienen. Singer (1962) gab in seinem Buch: "The Agaricales in modern taxonomy" eine erschöpfende Darstellung dieser Reihe, die er in 16 Familien gliederte. Im Schlüssel zu diesen Familien spielt die Struktur des hymenophoralen Tramas, also des die Lamellen oder Röhrchen bildenden Geflechtes eine wichtige Rolle. Bei seinen Studien über die Entstehungsweise der Fruchtkörper beschrieb REIJNDERS (1963) auch die Entwicklung und Struktur der Lamellen oder Röhrchen. Sowohl Singer wie Reijnders illustrierten ihre Beschreibungen mit zahlreichen Mikrofotographien, die jedoch, soweit sie den Bau des hymenophoralen Tramas betreffen, undeutlich und wenig instruktiv geblieben sind.

Das Ziel der vorliegenden Untersuchungen war daher, das die Lamellen oder Röhrchen bildende Hyphengeflecht bei reifen Fruchtkörpern eingehender zu untersuchen und darzustellen. Beschrieben und abgebildet werden senkrechte Querschnitte durch Lamellen oder durch das die Röhrchen bildende Trama von Fruchtkörpern mit sporenbildenden Hymenien. Bei den Agaricales werden die verschiedenen Teile der Fruchtkörper schon in frühen Stadien angelegt. Die Entfaltung und Vergrösserung der reifen Fruchtkörper erfolgt nicht durch eine Neubildung von Zellen oder durch ein Wachstum der Hyphen, sondern beruht entweder auf einer Zellvergrösserung oder auf einem Aufquellen der Zellwände. Vor allem die sich vergrössernden, aufquellenden Zellen können bestimmten Unterteilen der Lamellen angehören. Im allgemeinen befindet sich im Zentrum der Lamelle das aus senkrecht verlaufenden Hyphen bestehende, 
mediane Trama. Die sich beidseits nach aussen anschliessenden Hyphenpartien werden Hymenopodien genannt und diese gehen in das Subhymenium über. Diesem wiederum entspringen die das Hymenium bildenden Basidien. Das Hymenopodium ist oft reduziert oder lässt sich vom Subhymenium nicht deutlich scheiden. Die Basidien sind oft von ebenfalls dem Subhymenium entspringenden, sterilen Zellen, den Basidiolen umgeben. Häufig kommen auch dem Hymenopodium oder Trama entspringende und das Hymenium überragende Cystiden vor.

\section{Material und Methoden}

Ausser bei Strobilomyces floccopus wurde zum Anfertigen der Querschnitte durch das hymenophorale Trama stets frisches, im Herbst 1963 grösstenteils in den Niederlanden in der Umgebung von Breda gesammeltes Material verwendet. Soweit die Art nicht direkt erkannt wurde, wurde sie mit Hilfe der Bestimmungsbücher von KüHNER und Romagnesi (1953) oder von Moser (1955) determiniert. Untersucht wurden insgesamt mehr als hundert Arten. Beschreibungen und Abbildungen wurden vorläufig von 24 Arten ausgeführt, die als typische Vertreter der 16 von SINGER (1962) gegebenen Familien gelten können.

Die Querschnitte durch die Lamellen wurden stets senkrecht zum untern Lamellenrand ausgeführt; meist wurde hierzu ein Hutsegment mit 4-6 Lamellen herauspräpariert und auf einer Korkunterlage mit einem Rasiermesser geschnitten. Mit dem Gefriermikrotom ausgeführte Schnitte blieben meist unbefriedigend. Die brauchbaren Handschnitte hatten eine Dicke von 12-20 $\mu$. Diese wurden vorerst in Wasser oder wenn nötig in Lactophenol untersucht. Um die Zellwände und besonders die Schnallen besser sichtbar zu machen, wurden die Schnitte mit in Ammoniak gelöstem Kongorot gefärbt. Differenziert wurde in $70 \%$ Alkohol. In Lactophenol nimmt bei den mit Kongorot gefärbten Schnitten das Trama eine bläuliche und das Hymenium eine violette Farbe an.

Sowohl Zellwände wie Protoplasma und andere Zelleinschlüsse färben sich leicht mit dem in Wasser löslichen Cresylblau. Das Trama erhält meist eine rotviolette, das Hymenium eine dunkelblaue Farbe. Vakuolen, andere Zelleinschlüsse und Inkrustierungen der Wand lassen sich in mit Cresylblau gefärbten Präparaten leicht wahrnehmen. Gute Resultate lieferten sie auch für Untersuchungen der Basidiosporenwand. Präparate in Lactophenol wurden stets mit Baumwollblau und Trypanblau gefärbt und sind für längere Zeit haltbar. Zur Prüfung der Amyloidität der Hyphen, der Basidiosporen und deren Wandverdickungen wurde stets Melzer's Reagenz verwendet.

\section{Resultate}

1. Polyporus melanopus Sw. ex Fr. (Abb. 1)

Das Trama besteht aus einem dichten Geflecht von subregulär 
verlaufenden Hyphen. Meist verlaufen sie mehr oder weniger senkrecht von oben nach unten oder biegen etwas nach aussen gegen das Hymenium. Teilweise sind sie verhältnismässig dickwandig, englumig und die wenigen Querwände sind stets mit Schnallen versehen. Dünnere Hyphen verlaufen quer zu den dickeren und funktionieren als "Bindhyphen". Eine subhymeniale Schicht lässt sich vom Trama kaum abgrenzen. Sich vergrössernde Zellen kommen kaum vor und daher ist das Trama zähe und lederig. Die in Form und Grösse normalen, chiastischen Basidien bilden je vier längliche, fast zylinderische, glattwandige, farblose und nicht amyloide Basidiosporen.

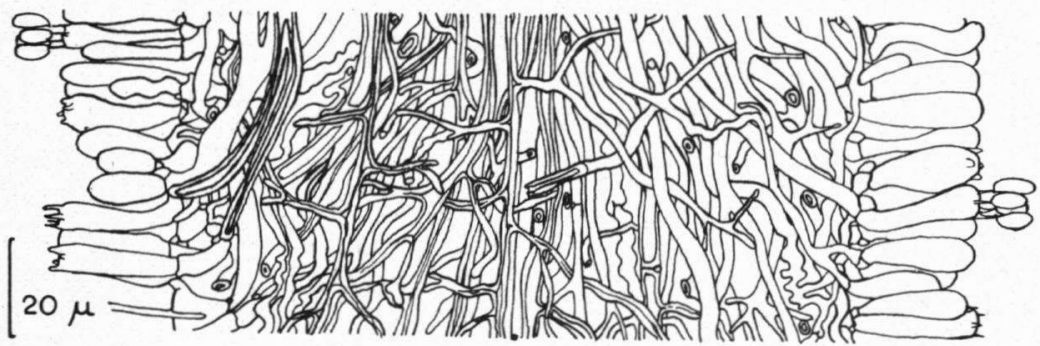

Abb. 1. Polyporus melanopus, Schnitt durch einen Teil des hymenophoralen Tramas.

Die Röhren sind an der rundlichen Mündung mit sterilen Hyphenhaaren besetzt. Im Hymenium fehlen dagegen Cystiden oder andere sterile Zellen.

Die Gattung Polyporus Mich. ex Fr. sensu stricto wurde von Singer (1962) auf Grund ihrer weitgehenden Übereinstimmung mit den typischen Vertretern der Gattung Pleurotus (Fr.) Kummer zu den Agaricales gestellt. Bei Pleurotus entsteht das Hymenium auf Lamellen und nicht in Röhrchen. Die Gattung Polyporus im Sinne von Singer umfasst nur noch wenige Arten. Die meisten der zum Beispiel von Overholts (1953) dazu gestellten Arten sind auszuschliessen und gehören zu den verschiedensten Gattungen und Familien der Aphyllophorales (vgl. Donk, 1933, 1964).

\section{Pleurotus dryinus (Pers. ex Fr.) Kummer (Abb. 2)}

Die Hyphen des Tramas sind gleich gebaut wie bei der oben beschriebenen Polyporus-Art und verlaufen ebenfalls regulär oder etwas irregulär. Ausser den dickwandigen kommen in geringer Zahl auch dünnwandige, schmälere oder breitere Hyphen vor, die teilweise grosse Vakuolen enthalten und sich mit Methylenblau violett färben. Die Art hat ein dickes, zelliges Subhymenium. Die Basidien und Basidiosporen stimmen mit denen von Polyporus melanopus weitgehend überein.

\section{Hygrocybe conica (Scop. ex Fr.) Kummer (Abb. 3)}

Das Trama dieser Art ist regulär gebaut. Die parallel in senkrechter Richtung verlaufenden Hyphen sind teilweise angeschwollen und 


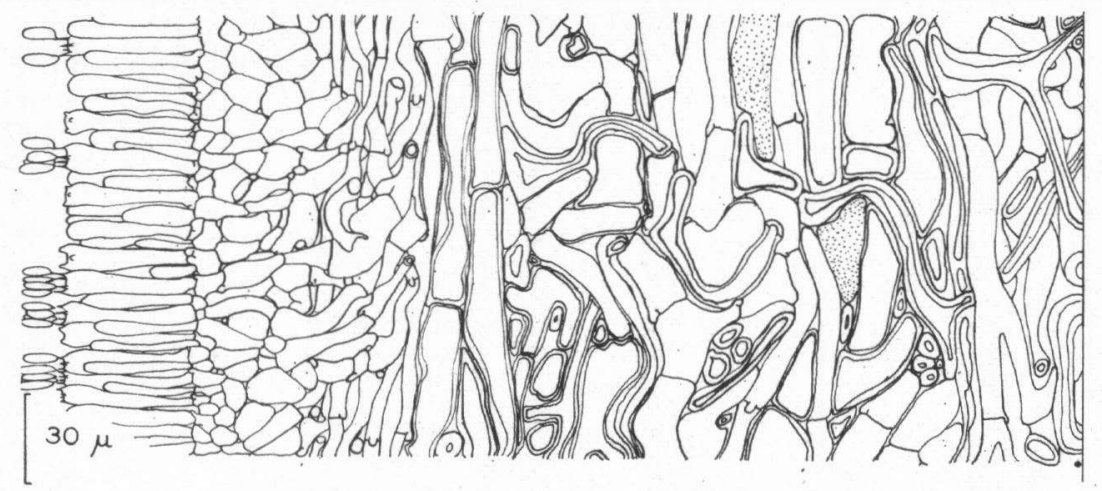

Abb. 2. Pleurotus dryinus, Schnitt durch eine Lamellenpartie.
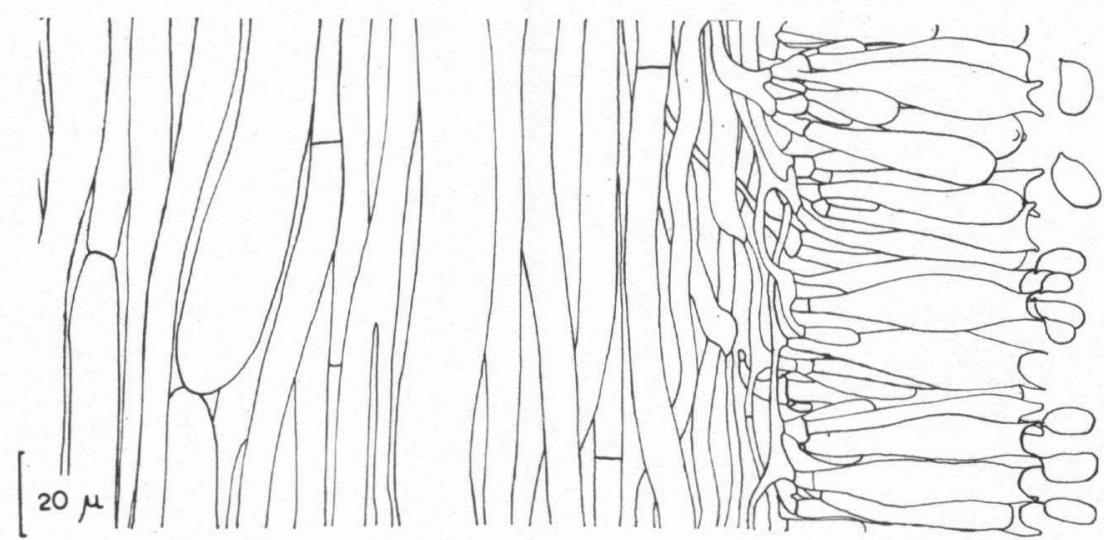

Abb. 3. Hygracybe conica, Schnitt durch einen Teil einer Lamelle.

bestehen aus grossen, bis zu $20 \mu$ breiten Zellen. Ihr Inhalt färbt sich mit Methylenblau und mit Cresylblau; Schnallen fehlen. Bei den untersuchten Fruchtkörpern trugen die grossen, vor allem auffallend langen Basidien stets nur je zwei Basidiosporen. Bei dieser parthenogenetischen Form sind auch die Hyphen haploid und darum fehlen Schnallen. Bei der dikaryotischen Form bilden die Basidien je vier Basidiosporen und die Hyphen sind stets mit Schnallen versehen. Die Basidiosporen sind farblos, nicht amyloid und glattwandig.

Die Gattung Hygrocybe (Fr.) Wünsche gehört zu den Hygrophoraceae, die sich vor allem durch gymnocarpe Fruchtkörper, breite, wachsige Lamellen und durch grosse, besonders lange Basidien auszeichnen. Bei den Vertretern der Gattung Hygrophorus Fr. ist das Trama im Gegensatz zu Hygrocybe bilateral gebaut; bei den Arten der Gattung Camarophyllus (Fr.) Karst. ist es unregelmässig (Moser, 1955). Auf Grund dieser und anderer Merkmale sieht man in den Hygrophoraceae oft die primitivsten Vertreter der Agaricales und die andern Familien werden von ihnen abgeleitet. 


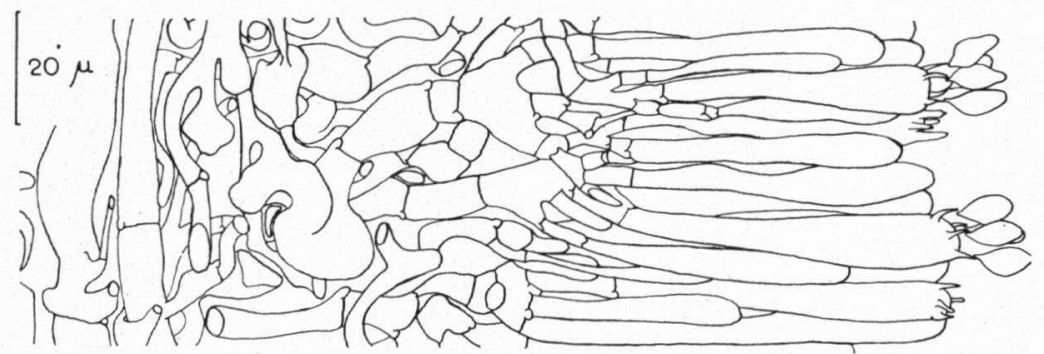

Abb. 4. Marasmius oreades, Schnitt durch einen Teil einer Lamelle.

4. Marasmius oreades (Bolt. ex Fr.) Fr. (Abb. 4)

Bei dieser Art ist das hymenophorale Trama irregulär und pseudoamyloid. Die Hyphen haben ziemlich dicke Wände und bei den Septen sind stets Schnallen vorhanden. Die Basidien sind sehr lang $(60-80 \mu)$ und erinnern an diejenigen der Hygrophoraceae. Auch die Lamellen sind ziemlich dick. Singer (1962) stellte Marasmius jedoch auf Grund anderer Merkmale zu den übrigens heterogene Elemente enthaltenden Tricholomataceae.

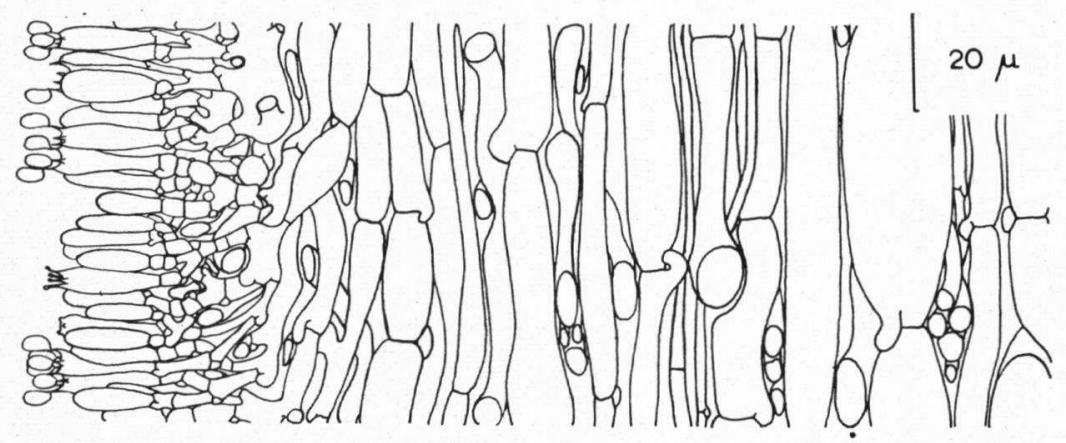

Abb. 5. Clitocybe nebularis, Schnitt durch einen Teil einer Lamelle.

\section{Clitocybe nebularis (Batsch ex Fr.) Kummer (Abb. 5)}

Das reguläre Trama besteht aus dünnwandigen, teilweise schmalen, teilweise angeschwollenen, stets mit Schnallen versehenen Hyphen. Meist verlaufen sie in vertikaler Richtung, teilweise aber auch horizontal und sind dann im Querschnitt durch die Lamelle durchgeschnitten. Das Subhymenium besteht aus kleinen, eckigen oder länglichen Zellen. Die etwas keuligen Basidien sind verhältnismässig klein. Die farblosen, nicht amyloiden Basidiosporen enthalten eine oder wenige, stark lichtbrechende Vakuolen. Die Art kann als typischer Vertreter der Tricholomataceae gelten.

6. Lentinellus cochleatus (Pers. ex Fr.) Karst. (Abb. 6)

Auch bei dieser Art ist das Trama der Lamellen regulär gebaut? Die mit Schnallen versehenen Hyphen haben aber dickere Wände 


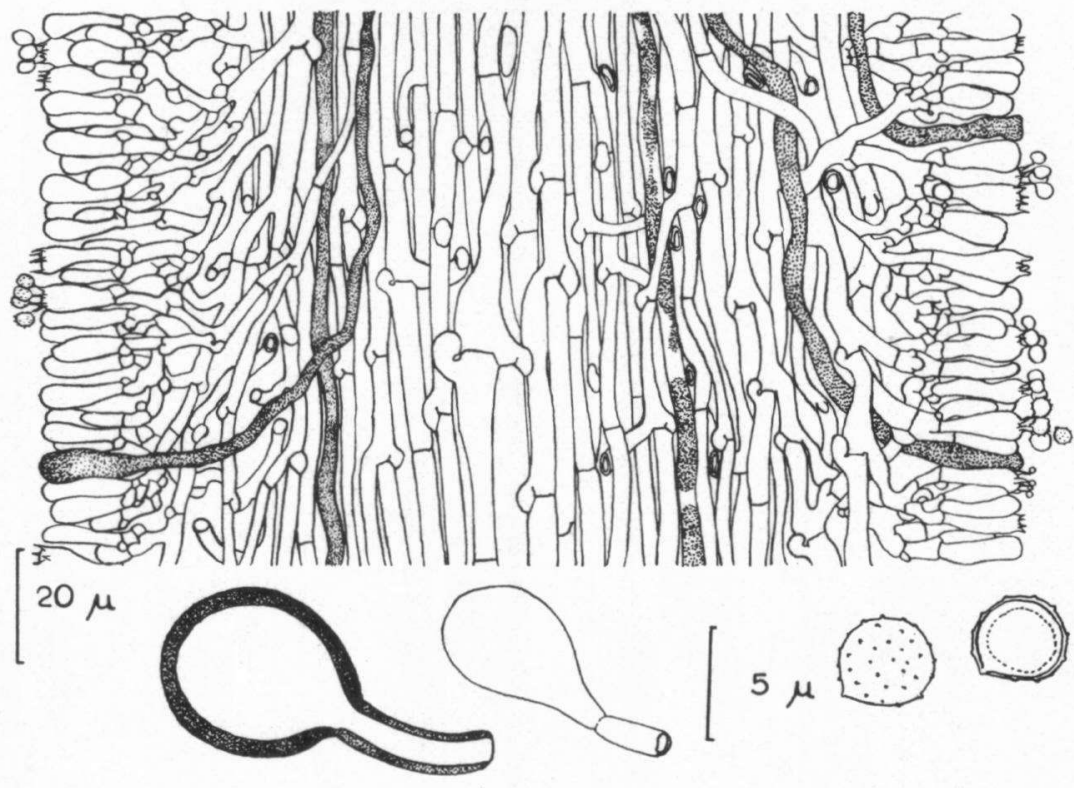

Abb. 6. Lentinellus cochleatus, Schnitt durch einen Teil einer Lamelle, unten links zwei Chlamydosporen, rechts zwei Basidiosporen.

und sind nicht angeschwollen. Im Trama verlaufen auch gelbliche, eine körnige oder ölige Masse enthaltende Hyphen. Sie sind kaum septiert und enden als "Gloeocystiden" im Hymenium. Ihr Inhalt lässt sich mit verschiedenen Reagenzien leicht färben. Die nur 4-5 $\mu$ grossen Basidiosporen sind ziemlich dickwandig, fein stachelig ornamentiert und stark amyloid.

Durch die Bildung von Gloeocystiden und durch die genannten Merkmale der Basidiosporen erinnert dieser Pilz nach RomagnesI (1953) an bestimmte Vertreter der Aphyllophorales, zum Beispiel an Auriscalpium vulgare S. F. Gray (Hydnum auriscalpium L. ex Fr.) und aus diesem Grunde stellte MaAs GeEsteranus (1963) die Gattung Lentinellus Karst. zu den Aphyllophorales in eine neue Familie, die er Auriscalpiaceae nannte. Die andern Gattungen dieser Familie bilden jedoch keine Lamellen, sondern das Hymenium entsteht auf stacheligen Fortsätzen. Die Stellung der Gattung Lentinellus ist demnach umstritten; von den Agaricales sollte sie jedoch ausgeschlossen werden.

\section{Amanita citrina (Schaeff.) Gray (Abb. 7)}

Nach den Angaben in der Literatur sollen sich bei den Amanitaceae die Lamellen durch den bilateralen Bau ihres Tramas auszeichnen. Bei Amanita citrina und andern untersuchten Amanita-Arten ist das Trama jedoch anders gebaut als zum Beispiel bei den Arten der Gattung Boletus oder bei Paxillus involutus (Abb. 19). Amanita citrina hat in der Lamellenmitte ein schmales, reguläres, aus schmalen 


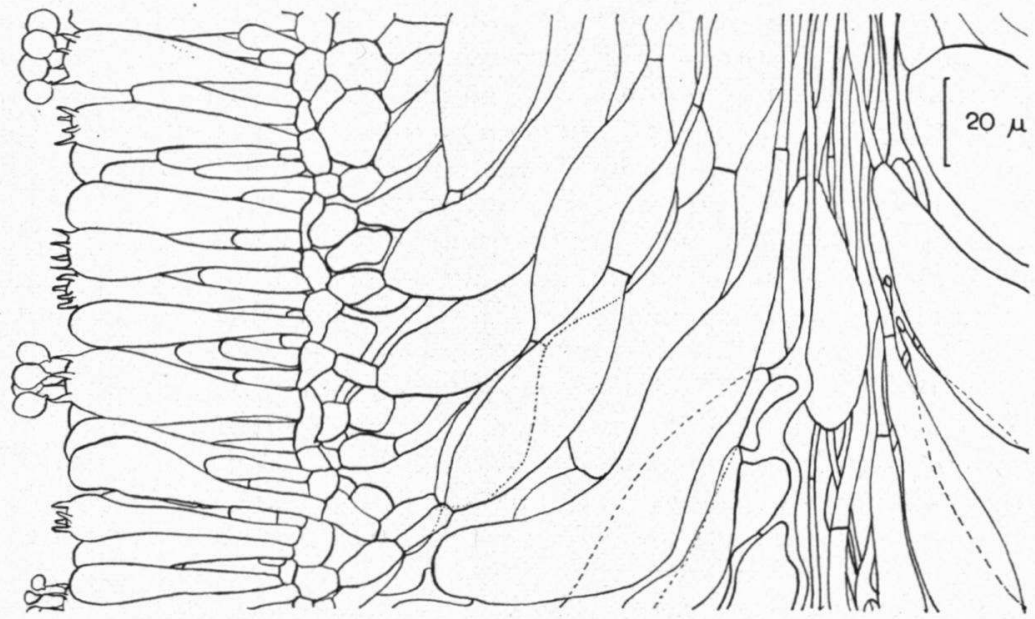

Abb. 7. Amanita citrina, Schnitt durch einen Teil einer Lamelle.

Hyphen bestehendes Trama. Beidseitig entwickelt sich auf diesem ein breites, aus stark angeschwollenen Zellen bestehendes Hymenopodium (Abb. 7). Das sich auf diesem erhebende Subhymenium besteht aus kurzen Hyphengliedern; Schnallen fehlen. Die grossen, ebenfalls angeschwollenen Basidien haben eine dünne Wand und bilden je vier verhältnismässig kleine, fast kugelige, glattwandige, farblose, amyloide Basidiosporen.

8. Pluteus atricapillus (Secr.) Sing. (Abb. 8)

Bei den von Singer (1962) zu den Amanitaceae gestellten Gattungen Pluteus Fr. und Volvariella Speg. haben die Lamellen ein invers ge-

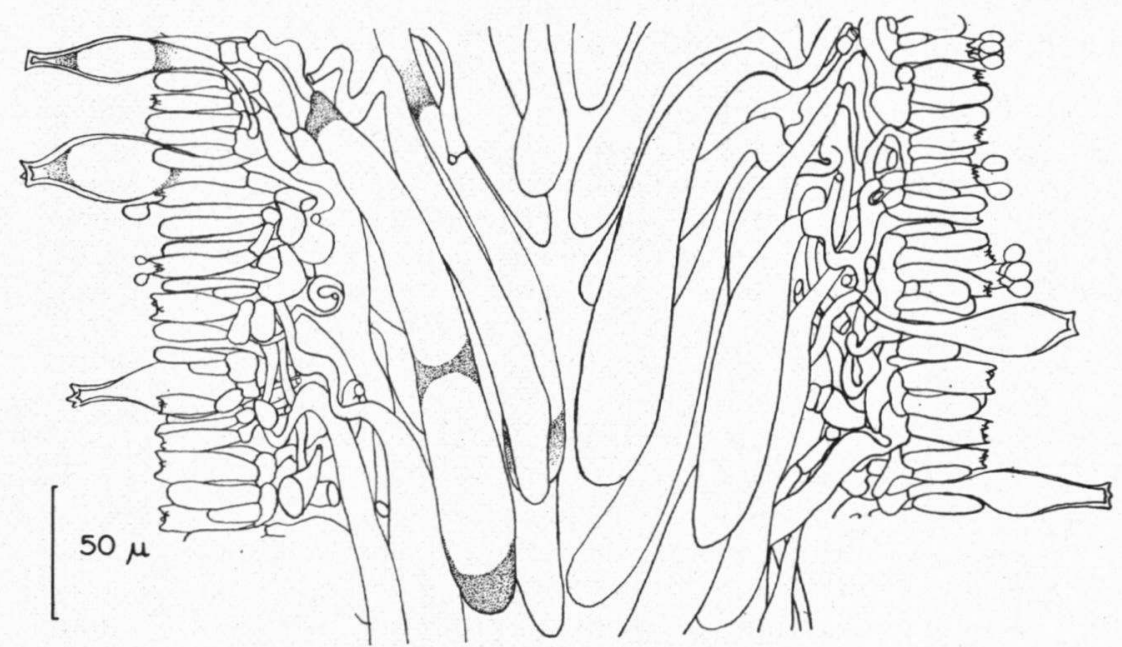

Abb. 8. Pluteus atricapillus, Schnitt durch einen Teil einer Lamelle. 
bautes Trama. Von den beiden Subhymenien aus verlaufen schräg nach innen lange, breite, angeschwollene, stark vergrösserte Zellen. Sie enthalten grosse Vakuolen und enden in der Mitte der Lamelle blind. Die Entstehungsweise invers gebauter Lamellen wurde zum Beispiel von ReIJNDERS (1963) beschrieben. Junge Lamellen sind sehr dünn und mehr oder weniger regulär gebaut. Im Gegensatz zu Amanita entwickeln sich die grossen Zellen nicht im Hymenopodium, sondern in der Lamellenmitte, wodurch das Trama auseinandergerückt wird. Durch die angeschwollenen Zellen erhalten die Lamellen ihre endgültige Form und sie enthalten die für die Bildung der Basidiosporen nötigen Reservestoffe.

Bei den untersuchten Pluteus-Arten konnten bei den Hyphen keine Schnallen beobachtet werden. Die Basidien sind von normaler Grösse oder eher klein und die glatten, farblosen, nicht amyloiden Basidiosporen bilden ein lachsfarbiges Pulver. Auffällig sind die charakteristisch gebauten Cystiden.

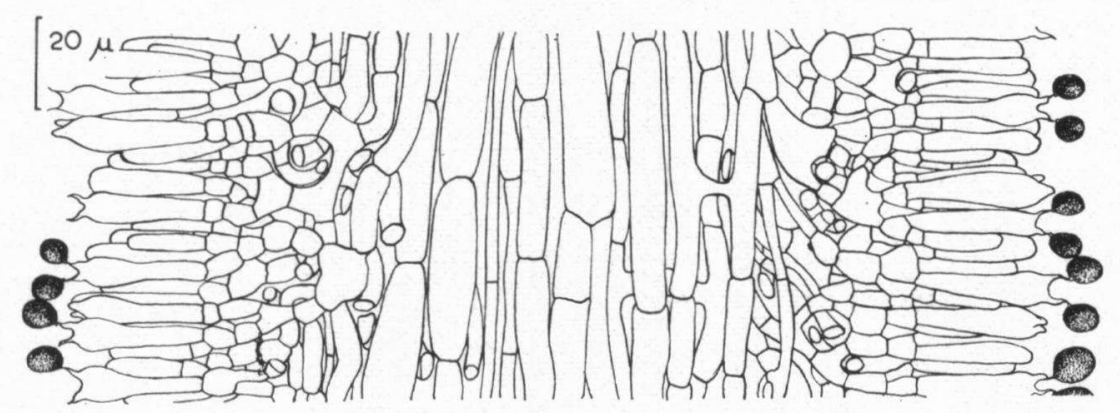

Abb. 9. Agaricus bisporus, Schnitt durch einen Teil einer Lamelle.

\section{Agaricus bisporus (Lange) Imbach (Abb. 9)}

Das Trama der Lamellen ist regulär gebaut. Die Hyphen sind dünnwandig und ohne Schnallen regelmässig septiert. Zwischen den parallel verlaufenden Hyphen kommen oft Anastomosen vor. Manche Hyphen bestehen aus stark verbreiterten Zellen. Die auf einem kleinzelligen Subhymenium entstehenden Basidien bilden auf charakteristisch gebogenen Sterigmen je zwei reif dunkle, doppelwandige, glatte Basidiosporen.

\section{Lepiota ignicolor Bres. (Abb. 10)}

Das reguläre Trama der reifen Lamellen besteht aus teilweise grossen, vor allem breiten, teilweise schmalen und langen Hyphengliedern. Schnallen sind regelmässig vorhanden und auch Anastomosen wurden beobachtet. Im aus rundlichen oder unregelmässigen Zellen bestehenden Subhymenium scheinen Schnallen zu fehlen. Die länglichen, stark asymmetrischen Basidiosporen sind hell und haben eine einfache, dünne Wand. 


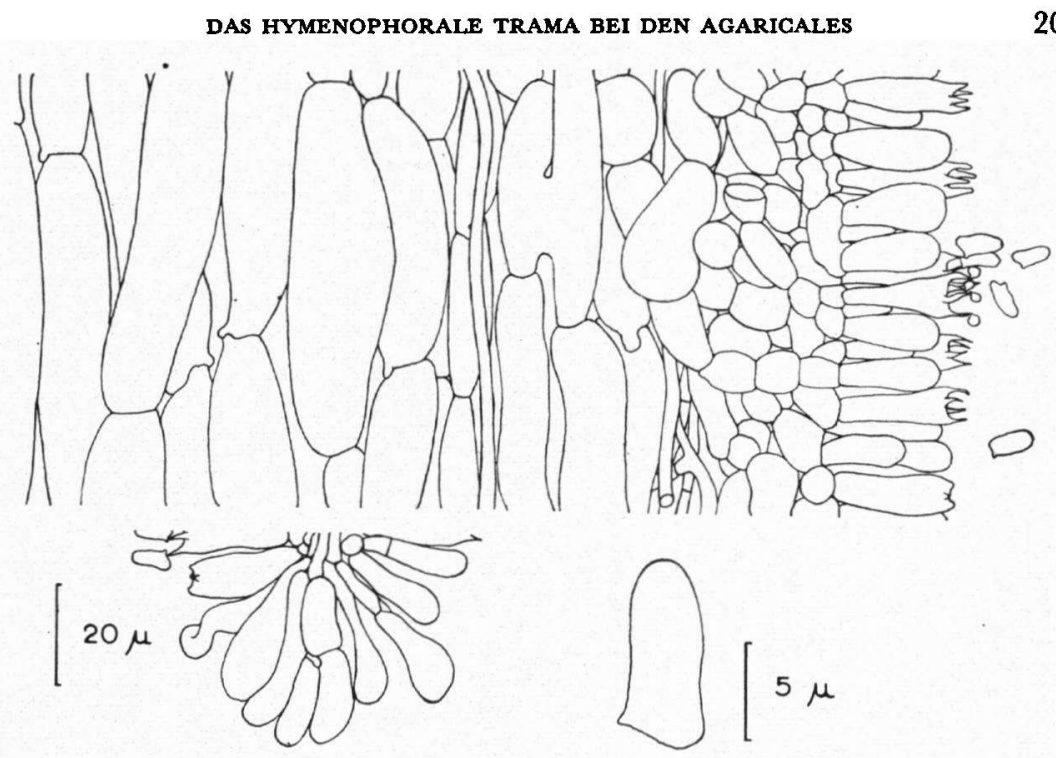

Abb. 10. Lepiota ignicolor, Schnitt durch einen mittleren Teil und durch den Rand einer Lamelle, rechts unten eine Basidiospore.

11. Coprinus atramentarius (Bull. ex Fr.) Fr. (Abb. 11)

Den dünnen Lamellen entsprechend ist das subregulär gebaute Trama sehr schmal. Die Hyphen haben dünne Wände und Schnallen sind nur ausnahmsweise vorhanden. Teilweise bestehen die Hyphen aus unregelmässig geschwollenen Zellen. Die Basidien stimmen in ihrem Bau weitgehend mit denen von Lepiota ignicolor überein: sie sind oben mehr oder weniger deutlich in die Sterigmen verbreitert. Die Basidien sind an der Basis von abgerundeten Zellen, den Basidiolen oder Pseudoparaphysen umgeben. Ferner finden sich die langen, cystidenartigen Zellen, durch die die zarten Lamellen gegenseitig auf Abstand gehalten werden. Die dunkeln, glattwandigen Basidiosporen haben einen apikalen Keimporus.

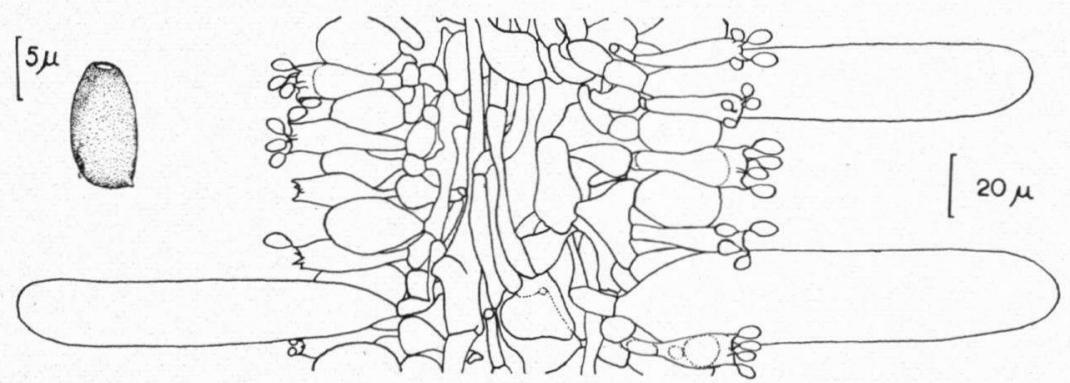

Abb. 11. Coprinus atramentarius, Schnitt durch einen Teil einer Lamelle, links oben eine Basidiospore. 


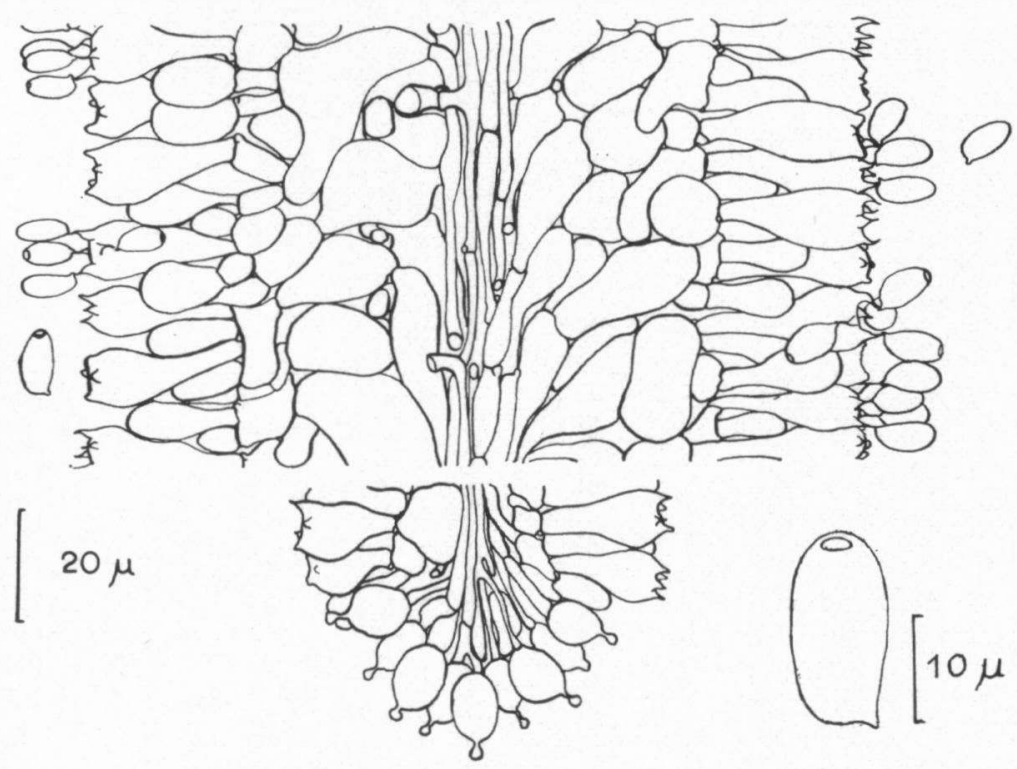

Abb. 12. Conocybe semiglobata, Schnitte durch einen mittleren Teil und durch den Rand einer Lamelle, rechts unten eine Basidiospore.

12. Conocybe semiglobata Kühn. \& Sing. (Abb. 12)

Die Gattung Conocybe Fayod gehört nach Singer (1962) in die Familie der Bolbitiaceae und diese soll den Coprinaceae nahe stehen. Conocybe semiglobata stimmt im Bau von Trama, Hymenium, Basidien und Basidiosporen auch weitgehend mit Coprinus überein. Der reguläre Teil des Tramas ist verhältnismässig schmal; die dünnwandigen Hyphen sind mit Schnallen versehen. Das Subhymenium besteht aus unregelmässig vergrösserten, rundlichen oder länglichen Zellen. Die Basidien sind keulig und die glattwandigen Basidiosporen haben apikal einen deutlichen Keimporus.

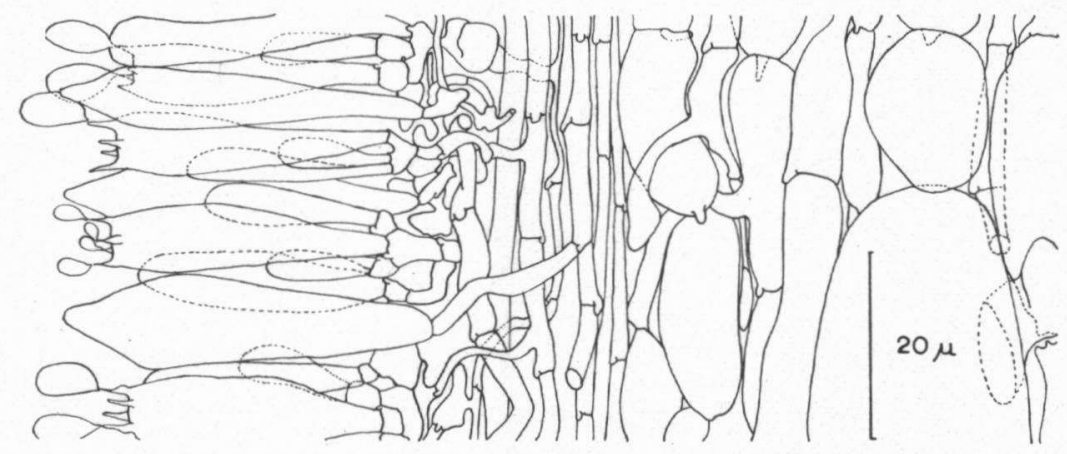

Abb. 13. Stropharia aeruginosa, Schnitt durch einen Teil einer Lamelle. 
13. Stropharia aeruginosa (Curt. ex Fr.) Quél. (Abb. 13)

Die Lamellen haben ein mehr oder weniger regulär gebautes Trama. Die in der Mitte verlaufenden Hyphen sind breit und bestehen aus oft stark angeschwollenen Zellen. Gegen den Rand sind die Hyphen schmäler; stets sind sie mit Schnallen versehen. Die von Cheilocystiden umgebenen Basidien entspringen einem kleinzelligen Subhymenium und sind in Form und Grösse normal. Bei den hellbraunen, glattwandigen Basidiosporen konnte kein Keimporus beobachtet werden.

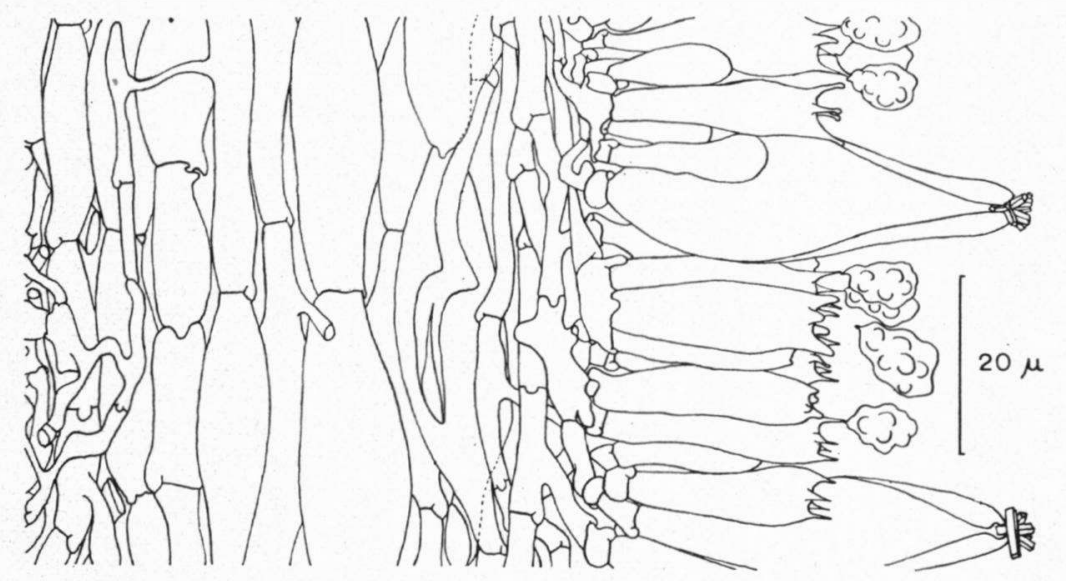

Abb. 14. Inocybe mixtilis, Schnitt durch einen Teil einer Lamelle.

\section{Inocybe mixtilis (Britz.) Sacc. sensu Kühner (Abb. 14)}

Die Lamellen haben ein regulär gebautes Trama. In der Mitte besteht es aus breiteren, nach aussen schmäler werdenden, regelmässig septierten und mit Schnallen versehenen Hyphen. Sie sind verhältnismässig zartwandig. Das Subhymenium besteht aus kriechenden Hyphen, auf denen die etwas keuligen Basidien entstehen. Die auch unter dem Mikroskop bräunlichen Basidiosporen sind höckerig warzig und ziemlich dickwandig. Auffällig sind ferner die bauchigen Gystiden (Metuloiden), deren sich mit Crresylblau violett färbende Wand nach oben stark verdickt ist. In ihrem Scheitel werden stäbchenförmige Kristalle von Kalziumoxalat ausgeschieden.

\section{Cortinarius anomalus Fr. (Abb. 15)}

Die Hyphen des regulär gebauten Tramas bestehen vor allem in der Lamellenmitte aus stark verbreiterten, kurzen Zellen. Die stets mit Schnallen versehenen Querwände sind oft etwas verdickt. Nach aussen geht das mediane Trama ohne scharfe Grenze in das aus kriechenden Hyphen bestehende Subhymenium über. Die Basidiosporen haben eine doppelte, fein ornamentierte, bräunliche Wand.

Die Gattungen Inocybe und Cortinarius gehören zu den vor allem durch makroskopische Merkmale charakterisierten Cortinariaceae. Die 


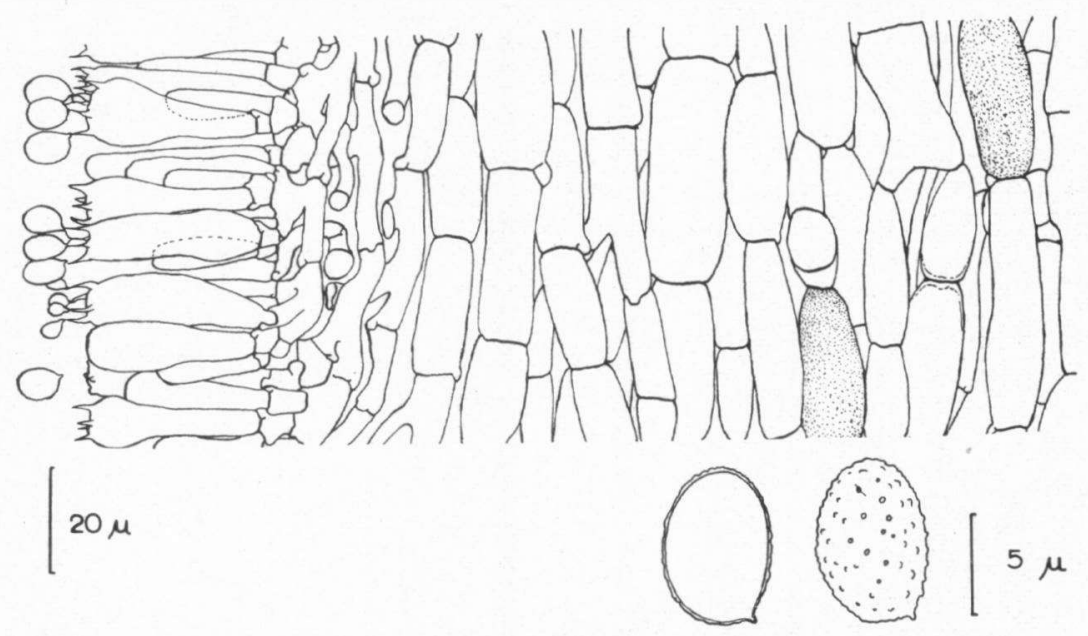

Abb. 15. Cortinarius anomalus, Schnitt durch einen Teil einer Lamelle, rechts unten zwei Basidiosporen.

beiden untersuchten Vertreter stimmen auch im Bau des hymenophoralen Tramas weitgehend miteinander überein.

16. Crepidotus variabilis (Pers. ex Fr.) Kummer (Abb. 16)

Singer (1962) stellte die Gattung Crepidotus (Fr.) Quél. in eine eigene Familie der Crepidotaceae, die sich jedoch von den Cortinariaceae
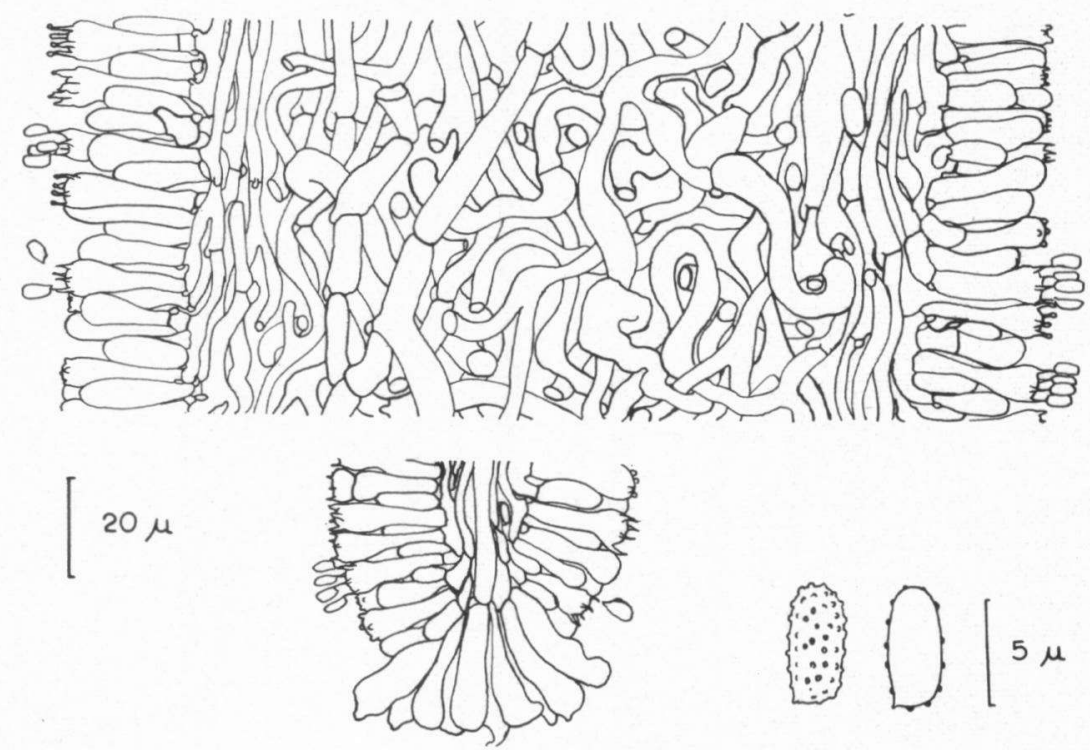

Abb. 16. Crepidotus variabilis, Schnitte durch einen mittleren und den untern Teil einer Lamelle, rechts unten zwei Basidiosporen. 
nur durch untergeordnete Merkmale unterscheiden lässt. Bei Crepidotus variabilis ist das Trama der Lamellen im Gegensatz zu den beiden oben abgebildeten Vertretern der Cortinariaceae typisch unregelmässig, "irregulär" gebaut und besteht aus in allen Richtungen verlaufenden, verflochtenen, dünnen oder etwas angeschwollenen Hyphen. Diese sind oft nur wenig septiert; bei den Querwänden sind jedoch stets Schnallen vorhanden. Gegen das Subhymenium sind die Hyphen dünner und verlaufen dann mehr oder weniger parallel zur Oberfläche. Die unter dem Mikroskop farblosen Basidiosporen bilden im Gegensatz zu denen der Cortinariaceae kein braunes, sondern ein hell braungelbes Pulver. Sie sollen eine zweischichtige Wand haben, was bei der untersuchten Art jedoch nicht mit Sicherheit festgestellt werden konnte. Wohl konnte eine feine Ornamentierung beobachtet werden.

\section{Clitopilus prunulus (Scop. ex Fr.) Kummer (Abb. 17)}

Diese und die folgende Art gehören in die Familie der Rhodophyllaceae und zeichnen sich durch ein rötliches Sporenpulver und durch meist eckige Basidiosporen aus.

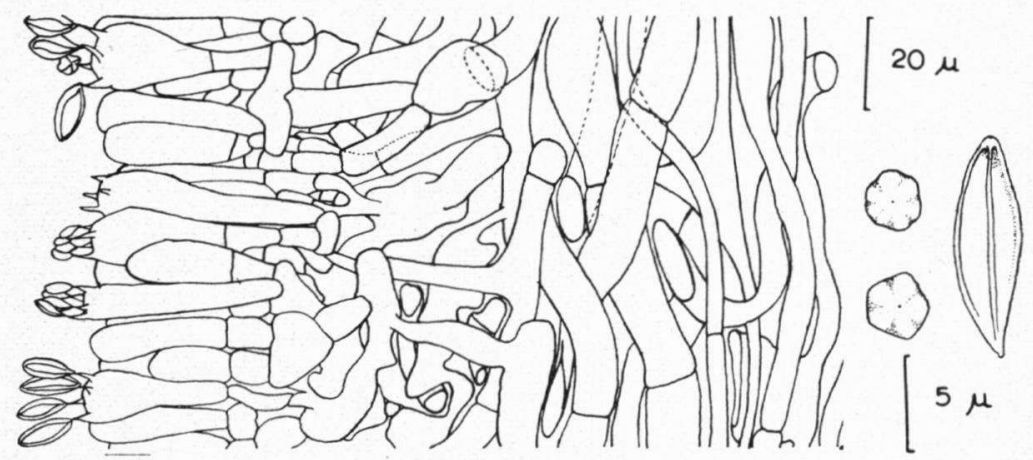

Abb. 17. Clitopilus prunulus, Schnitt durch einen Teil einer Lamelle, rechts drei Basidiosporen, von der Seite und von oben gesehen.

Bei Clitopilus prunulus ist das Trama in der Lamellenmitte noch einigermassen regulär (subregulär) gebaut und die schnallenlosen Hyphen bestehen aus breiten und langen Zellen. Das stark entwickelte Hymenopodium besteht aus nach aussen weichenden Hyphen, das schmale Subhymenium aus kurzen, abgerundet eckigen Zellen, denen je eine Basidie aufsitzt. Die grossen, spindelförmigen Basidiosporen sind in der Längsrichtung gefurcht und von oben gesehen 5-6-eckig.

18. Rhodophyllus sericeus (Bull. ex Fr.) Quél. (Abb. 18)

Das reguläre Trama besteht aus breiten und sehr langen, angeschwollenen, schnallenlosen Hyphengliedern. Dagegen sind im Subhymenium und vor allem an der Basis der breit keuligen Basidien Schnallen vorhanden. Die eckigen Basidiosporen haben einen deutlichen Apikulus und sind nicht amyloid. 


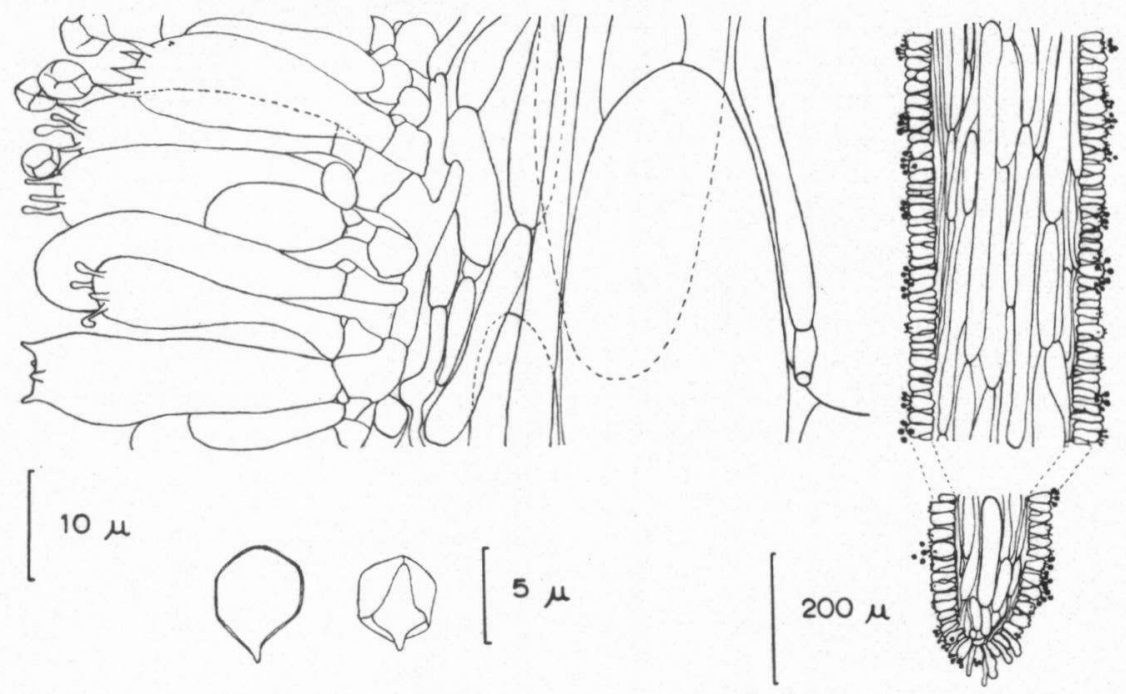

Abb. 18. Rhodophyllus sericeus, Schnitte durch eine Lamelle, sowie zwei Basidiosporen.

19. Paxillus involutus (Batsch ex Fr.) Fr. (Abb. 19)

Die Paxillaceae bilden zusammen mit den Boletaceae eine natürliche Einheit und zeichnen sicb zum Beispiel durch den Besitz eines bilateralen Tramas aus. Ferner fehlen angeschwollene Zellen und die Lamellen- oder Röhrenschicht lässt sich leicht vom Hut lösen.

Bei Paxillus involutus besteht das Trama der Lamellen aus schmalen, in der Mitte senkrecht, gegen den Rand kontinuierlich nach aussen verlaufenden Hyphen. Bei den Querwänden sind stets Schnallen vorhanden. Im Subhymenium verzweigen sich die Hyphen und bilden in Form und Grösse normale Basidien. Die glatten Basidiosporen haben eine ziemlich dicke, hellbraune Wand.

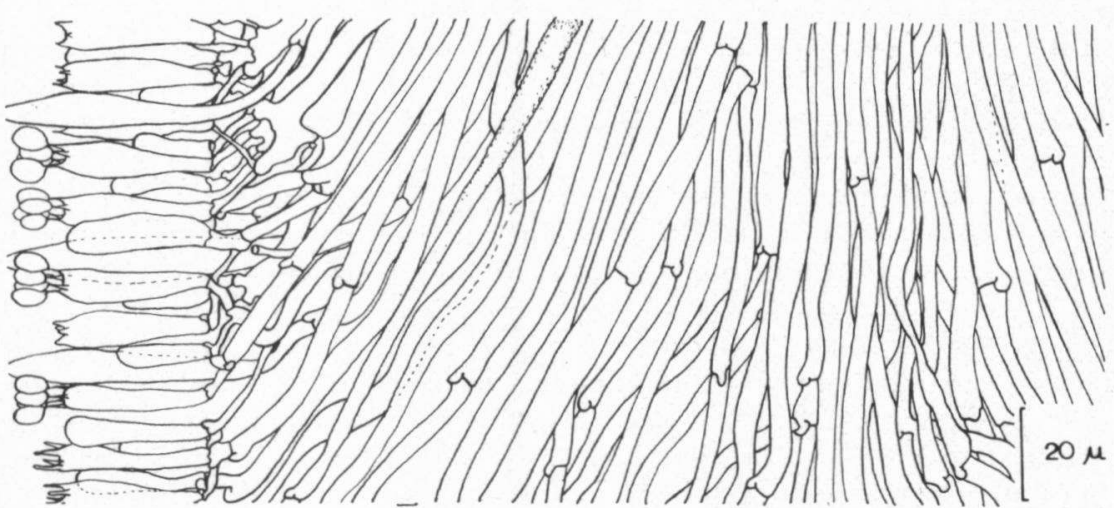

Abb. 19. Paxillus involutus, Schnitt durch einen Teil einer Lamelle. 
Früher sah man in Paxillus involutus eine Utbergangsform von den mit Lamellen versehenen Agaricales zu den sich durch Röhren auszeichnenden Boleten. In Wirklichkeit hat Paxillus bei den bisher besprochenen Agaricales keine nahen Verwandten.

\section{Suillus bovinus (L. ex Fr.) O. Kuntze (Abb. 20)}

Bei diesem typischen Vertreter der Boletaceae ist das Trama bilateral gebaut. Zwischen den locker verlaufenden, einer Schleimmasse eingebetteten Hyphen kommen Anastomosen vor. Die Hyphenwände sind nach aussen unscharf begrenzt und gehen in die Schleimmasse über; diese geht anscheinend aus den aussen verquellenden Hyphenwänden hervor. Angeschwollene Zellen kommen dagegen nicht vor. Das Hymenium besteht aus kleinen Basidien und aus Gruppen von an der Basis inkrustierten Cystiden.

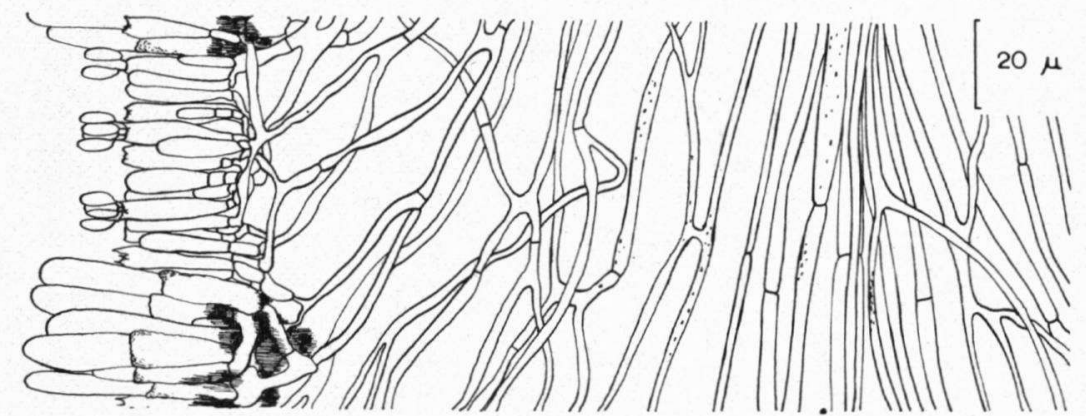

Abb. 20. Suillus bovinus, Schnitt durch einen Teil des hymenophoralen Tramas.

21. Gomphidius rutilus (Schaeff. ex Fr.) Lundell \& Nannf. (Abb. 21) Die Lamellen sind verhältnismässig dick. In ihrem Trama verlaufen nur noch einzelne Hyphen in senkrechter Richtung. Die reife Lamelle besteht aus einem unregelmässigen, hymenopodialen $\mathrm{Ge}-$ flecht von dicken, rauhwandigen, verschleimenden, oft inkrustierten Hyphen. An diesen entstehen grosse, zylinderische, teilweise ebenfalls inkrustierte Cystiden. Die einem zelligen Subhymenium aufsitzenden Basidien bilden auf kurzen Sterigmen je vier längliche, ein dunkles Pulver bildende Basidiosporen.

\section{Strobilomyces floccopus (Vahl ex Fr.) Karst. (Abb. 22)}

Da das Hymenium in Röhren entsteht, wurde die Gattung Strabilomyces Berk. bisher meist bei den Boletaceae untergebracht; SINGER (1962) und andere stellten sie in eine eigene Familie. Bei Strobilomyces floccopus = Strobilomyces strobilaceus (Scop. ex Fr.) Berk. ist das Trama bilateral gebaut. Die sich regelmässig verzweigenden Hyphen bestehen aus langen Zellen und haben keine Schnallen. Ausser farblosen kommen auch Hyphen mit einem dunkelbraunen Inhalt vor. Dem schmalen Subhymenium sitzen bauchige Basidien und Basidiolen auf. Ferner entspringen ihm in Form und Pigmentierung verschieden- 

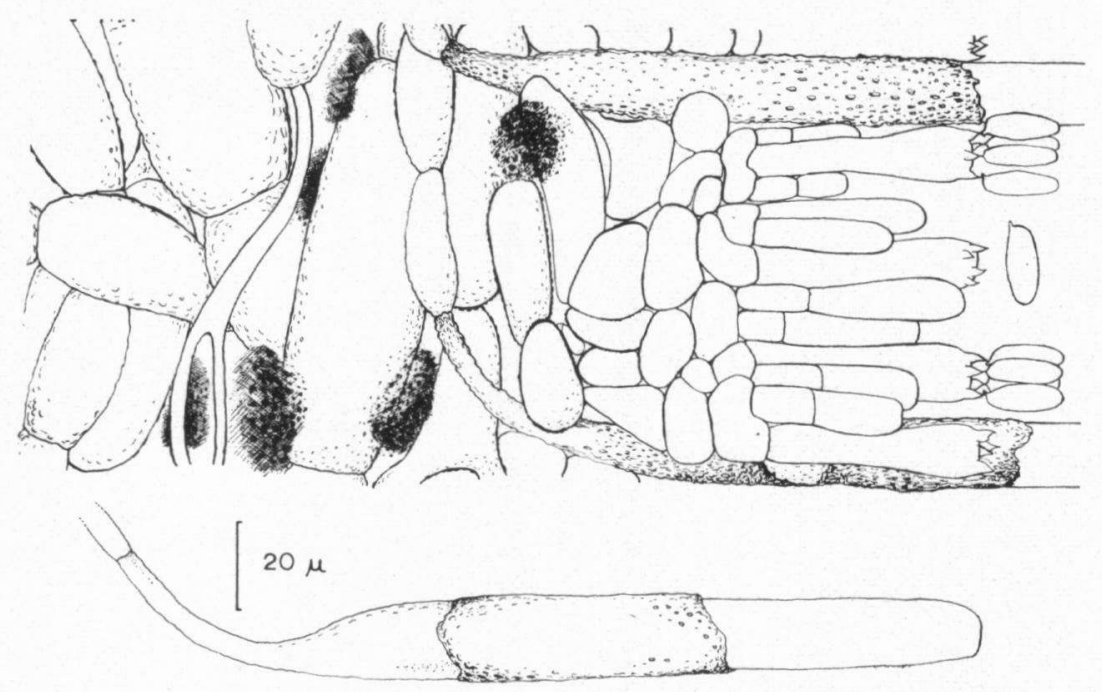

Abb. 21. Gomphidius rutilus, Teil einer Lamelle im Schnitt, sowie frei präparierte Cystide.

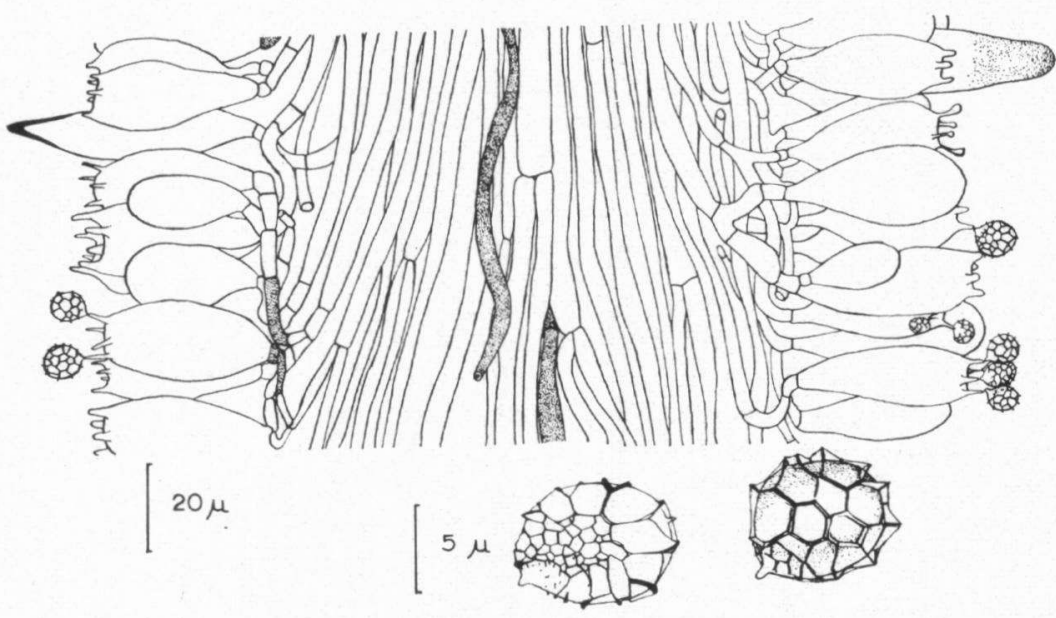

Abb. 22. Strobilomyces flcccopus, Schnitt durch einen Teil des die Röhren bildenden Geflechtes, unten zwei Basidiosporen.

artige Cystiden. Die breit ellipsoidischen Basidiosporen haben eine dunkle, ockergelbe Farbe und ihre Wand ist mit rotbraunen Leisten besetzt.

23. Russula soraria (Fr.) Romell (Abb. 23)

Bei dieser Art ist das Trama gemengt, es besteht aus verschiedenartigen Hyphenelementen. Ausser dünnwandigen, schmalen, schnal- 
lenlosen Hyphen kommen zahlreiche, kugelige Zellen, sogenannte Sphaerocysten vor. Diese liegen meist in Gruppen oder in kurzen Ketten. Ferner finden sich Hyphen mit einem stark lichtbrechenden Inhalt, der sich mit Cresylblau tief blau färbt. Diese Hyphen enden im Hymenium als Gloeocystiden. Das Subhymenium besteht aus kurzen Hyphengliedern. Ausser von Gloeocystiden sind die Basidien von Basidiolen und von grösseren Cystiden (Makrocystiden) umgeben. Die breit ellipsoidischen, mit einem deutlichen Apiculus versehenen Basidiosporen haben eine hyaline Wand mit amyloiden, sich mit Melzer's Reagenz blauschwarz färbenden, unregelmässigen, leistenförmigen Verdickungen.

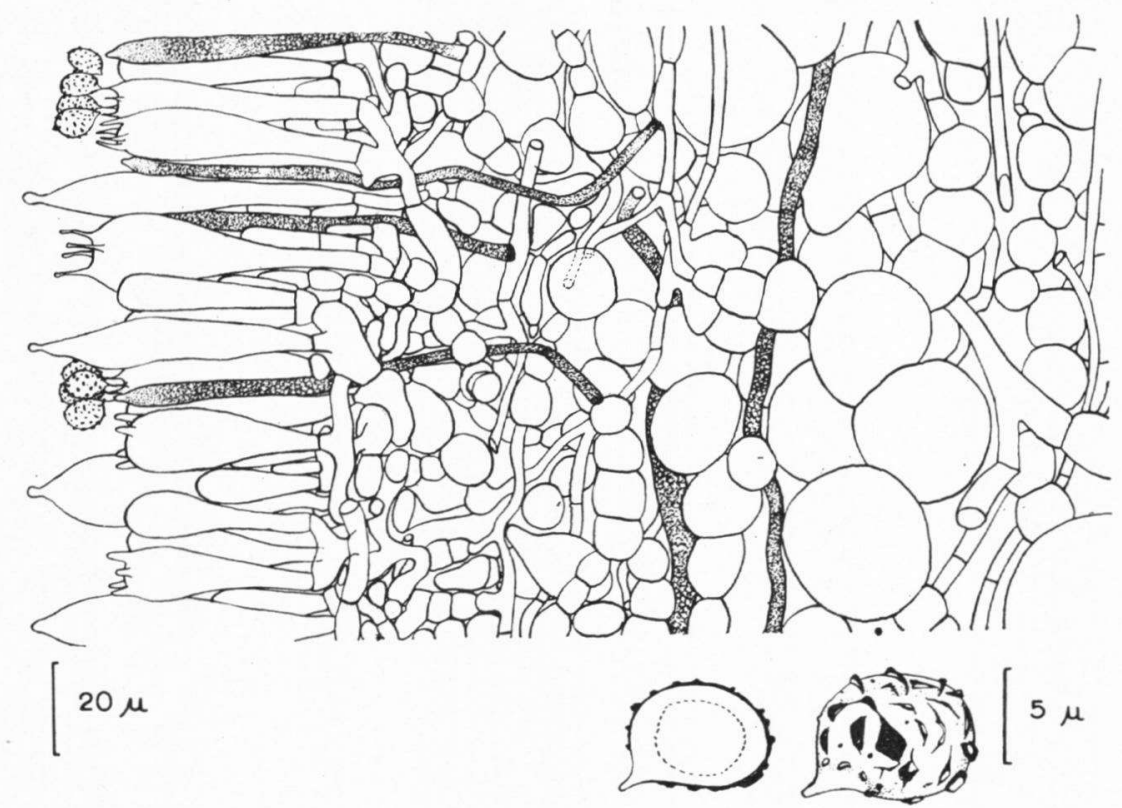

Abb. 23. Russula soraria, Schnitt durch einen Teil einer Lamelle, unten zwei Basidiosporen.

Die Gattungen Russula (Pers. ex Fr.) S. F. Gray und Lactarius DC. ex S. F. Gray bilden nach Singer und SMith (1960) zusammen mit einigen bisher bei verschiedenen Familien der Gastromycetes untergebrachten Pilzen eine natürliche Einheit, die astrosporoide Serie. Formen mit übereinstimmenden, mikroskopischen Merkmalen finden sich auch bei den Aphyllophorales.

\section{Lactarius necator (Bull. ex Fr.) Karst. (Abb. 24)}

Das Trama der Lamellen ist mehr oder weniger deutlich regulär gebaut. Sphaerocysten konnten nur im Hutgeflecht und nicht in den Lamellen beobachtet werden. Die Hyphen bestehen teilweise aus breiteren, teilweise aus schmäleren, meist kurzen Gliedern. Die 
Milchsaft enthaltenden Hyphen sind kaum oder nur wenig septiert und enden oft im Hymenium. Dieses besteht aus Basidien, Basidiolen und spitzlichen Cystiden. Die breit ellipsoidischen Basidiosporen sind durch die amyloiden Wandverdickungen auffällig ornamentiert.

\section{Besprechung Der Resultate}

Im Baue des hymenophoralen Tramas zeigen die Agaricales eine grosse Verschiedenheit. Die verschiedenen Bautypen gehen ausser mit makroskopischen Merkmalen auch mit andern mikroskopischen Merkmalen parallel, zum Beispiel mit dem Vorhandensein oder Fehlen von Schnallen, Cystiden, Gloeocystiden oder Basidiolen oder mit bestimmten Merkmalen der Basidien und Basidiosporen.

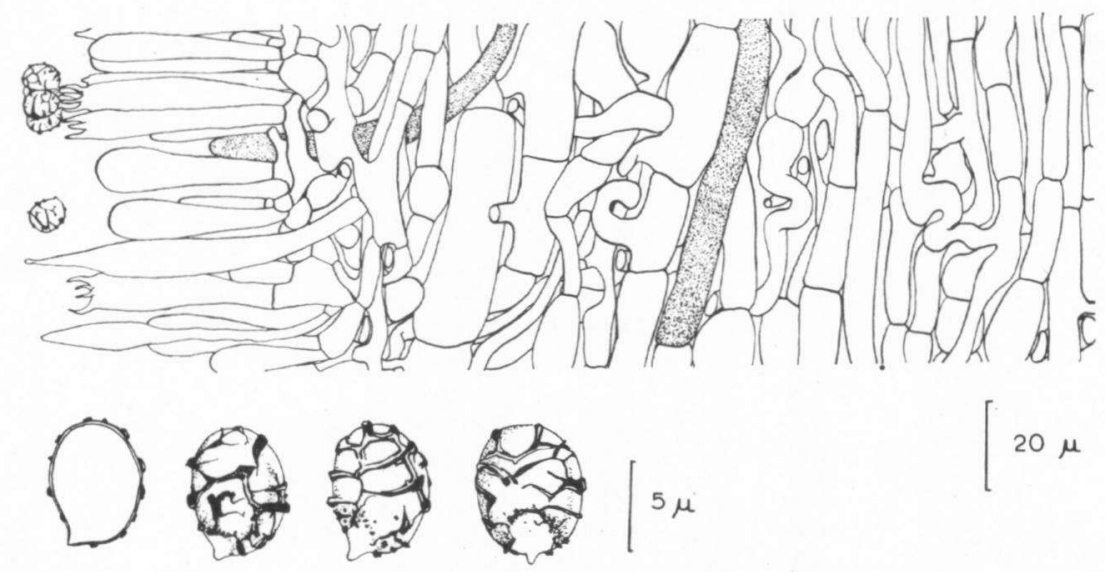

Abb. 24. Lactarius necator, Schnitt durch einen Teil einer Lamelle, unten vier Basidiosporen.

Aus verschiedenen Bauelementen besteht das Trama bei den Vertretern der Gattungen Lactarius, Russula und Lentinellus. Parallel damit geht eine Ornamentierung der Basidiosporen und eine Amyloidität der Wandverdickungen oder der gesamten Sporenwand. Lactarius und Russula werden schon lange zusammen mit einigen Gattungen mit angiocarpen Fruchtkörpern in eine eigene Familie gestellt (MaLENçon, 1931; Heim, 1948; Singer und Smith, 1960). Dagegen wurde Lentinellus noch von Singer (1962) zu den Tricholomataceae gestellt, während die Gattung nach Romagnesi (1953) und MaAs Geesteranus (1963) mit Auriscalpium vulgare und andern hydnoiden Vertretern der Aphyllophorales verwandt sein soll. Die letztgenannte Ansicht ist ohne Zweifel die richtige und diese Pilze gehören zusammen mit den Stereaceae im Sinne von Lemke (1964) ebenfalls in den weiteren Verwandtschaftskreis der Russulaceae.

Alle anderen Vertreter der Agaricales haben ein nicht gemengtes, homomeres Trama. Typisch bilateral gebaut ist es bei den untersuchten Vertretern der Paxillaceae, Boletaceae und Strobilomycetaceae. Es besteht aus schmalen, nach aussen verschleimenden Hyphen, 
dagegen fehlen grosse, geschwollene Zellen. Diese einander nahstehenden Familien haben nach LANGE und HaNSEN (1954) verwandtschaftliche Beziehungen zu den Aphyllophorales und können von diesen hergeleitet werden.

Bei den meisten andern Vertretern der Agaricales erhalten die Lamellen ihre endgültige Grösse durch ein Anschwellen bestimmter Zellen oder Hyphen des hymenophoralen Tramas. Charakteristisch gelagert sind diese angeschwollenen Zellen vor allem bei den Amanitaceae, deren Trama bisher als bilateral oder invers bezeichnet wurde. Bei den Vertretern der Gattung Amanita ist das Trama in jungen Stadien regulär gebaut. Bei der Entfaltung der Fruchtkörper schwellen dann die hymenopodialen, das innere Trama mit dem Subhymenium verbindenden Hyphenglieder auf. Der Bau einer derartigen Lamelle ist verschieden von dem einer typisch bilateral gebauten zum Beispiel von Paxillus. Er kann als pseudobilateral bezeichnet werden. Bei Pluteus und Volvariella befinden sich die aufschwellenden Hyphenzellen in der Mitte des Tramas, dessen regulärer Teil dadurch in zwei Schichten gespalten wird, zwischen denen sich die ebenfalls zwei Schichten bildenden "Schwellzellen" befinden. Ein derartiges Trama wird invers genannt.

Bei den untersuchten Arten der Gattungen Hygrocybe, Clitocybe, Agaricus, Lepiota, Stropharia, Cortinarius, Inocybe und Rhodophyllus vergrössern sich bei der Entfaltung der Fruchtkörper vor allem die mittleren Hyphenglieder des regulär gebauten Tramas. Ähnlich verhalten sich die Formen mit einem irregulären Trama, zum Beispiel Crepidotus variabilis oder Marasmius oreades, nur ist dann die Vergrösserung der Hyphenglieder nicht so deutlich. Coprinus atramentarius (Coprinaceae) und Conocybe semiglobata (Bolbitiaceae) haben verhältnismässig dünne Lamellen. Die Hyphen des medianen Tramas sind bleibend dünn, dagegen schwellen die kurzen Zellen des Subhymeniums und werden oft mehr oder weniger rundlich.

Wiederum anders gebaut ist das hymenophorale Trama bei den untersuchten Vertretern der Gattungen Polyporus und Pleurotus. Hier besteht das Trama aus einem dichten Geflecht von dickwandigen Hyphen, die von zarteren Bindhyphen umwunden werden. Dagegen fehlen sich vergrössernde Hyphenglieder oder Hyphen mit verquellenden Wänden. Nach der Struktur des Tramas beurteilt und auch auf Grund der nicht fleischigen, sondern lederigen Beschaffenheit der Fruchtkörper finden die beiden Gattungen bei den Aphyllophorales einen besseren Platz als bei den Agaricales. Bei den letzteren haben sie keine näheren Verwandten. Zu den Aphyllophorales zu stellen ist nach DoNk (1964) auch die Gattung Schizophyllum Fr., von der im Rahmen dieser Arbeit keine Vertreter untersucht wurden. Aber auch die untersuchte Art der Gattung Crepidotus zeigt in der Struktur des hymenophoralen Tramas und in der Konsistenz und Form der Fruchtkörper Anklänge an die Aphyllophorales.

Auf Grund der nur wenigen, im Rahmen dieser Arbeit untersuchten Arten lassen sich natürlich keine weitgehenden Schluss- 
folgerungen über die systematische Gliederung dieser Pilze ziehen. Es konnte jedoch bestätigt werden, dass die Boletaceae, die Paxillaceae, die Strobilomycetaceae und auch die Gomphidiaceae innerhalb der Agaricales eine natürliche Einheit darstellen. Mikroskopisch zeichnen sie sich durch ein bilaterales Trama und durch verschleimende Hyphenwände aus. Sie können in einer eigenen Subordnung der Boletineae zusammengefasst werden.

Die zu der Subordnung der Agaricineae zu stellenden Familien haben Vertreter, die sich mikroskopisch durch ein regulär, subregulär, pseudobilateral oder invers gebautes Trama auszeichnen. Dieses schliesst stets vergrösserte, angeschwollene, "aufgeblasene" Zellen ein.

Die Russulineae haben ein gemengtes, aus verschiedenartigen Hyphenelementen bestehendes Trama, sowie breite Basidiosporen mit amyloiden Wänden oder Wandverdickungen. Ihre nächsten Verwandten finden sich unter den Aphyllophorales bei den Stereaceae. In diesen Verwandtschaftskreis gehören auch Lentinellus und andere, vor allem hydnoide Gattungen. Wieder zu den Aphyllophorales zu stellen sind auch die Polyporaceae sensu Singer und wahrscheinlich auch die Crepidotaceae.

Die verschiedenen Ordnungen und Unterordnungen lassen sich dann durch folgende Merkmale auseinanderhalten:

Fruchtkörper reif fleischig oder brüchig, Trama angeschwollene oder nach aussen verschleimende Hyphenzellen einschliessend, Hymenophor meist aus Röhren oder Lamellen bestehend. . . . . Agaricales

Trama bilateral, aus zarten, nach aussen verschleimenden Hyphen bestehend, angeschwollene Zellen fehlen . . . . .Boletineae Trama aus teilweise angeschwollenen Hyphengliedern bestehend Trama homomer, mit teilweise angeschwollenen Hyphengliedern. . . . . . . . . . . . . Agaricineae Trama heteromer, Sphaerocysten oder Milchsaftzellen einschliessend, Basidiosporen mit amyloiden Wandverdickungen oder Wänden . . . . . . . . . . . . Russulineae Fruchtkörper häutig, lederig, holzig oder korkig, Trama ohne angeschwollene oder nach aussen verschleimende Hyphenzellen; Hymenophor glatt oder mit Zähnen, Waben, Röhren oder Lamellen

\section{ZUSAMMENFASSUNG}

Die Agaricales wurden von Singer (1962) neu umschrieben und auf 16 Familien verteilt. Im Rahmen dieser Arbeit wurde von diesen Familien von wenigstens einem Vertreter das hymenophorale Trama (Lamellen oder Röhren) anhand von senkrechten Querschnitten mikroskopisch untersucht und abgebildet. Bei den typischen Vertretern der Agaricales ist dieses stets mehr oder weniger deutlich regulär oder bilateral gebaut und schliesst bei reifen Stadien stets angeschwollene, vergrösserte Zellen ein. Bei den Boletaceae, den Strobilomycetaceae, den Gomphidiaceae und den Paxillaceae sind die Zell- 
wände nach aussen schleimig aufgequollen. Bei den Vertretern der Gattungen Polyporus, Pleurotus (Polyporaceae sensu Singer), Lentinellus und Crepidotus fehlen angeschwollene Zellen; die Hyphen sind dickwandig und bilden ein dichteres Geflecht. Diese und andere Gattungen, zum Beispiel Schizophyllum, werden daher besser zu den Aphyllophorales gestellt.

\section{LITERATUR}

Donk, M. A. 1933. Revision der Niederländischen Homobasidiomycetae Aphyllophoraceae II. Meded. Bot. Mus. Utrecht 9: 1-278.

1964. A conspectus of the families of Aphyllophorales. Persoonia 3: 199-324.

HeIm, R. 1948. Phylogeny and natural classification of macrofungi. Trans. Brit. Myc. Soc. 30: 161-178.

KühneR, R. und H. Romagnest. 1953. Flore analytique des Champignons supérieurs. Masson, Paris, $557 \mathrm{~S}$.

Lange, M. und L. Hansen. 1954. The phylogenetic position of Agaricales. Bot. Tidsskr. 51 : 185-194.

Lemke, P. A. 1964. The genus Aleurodiscus in North America. Canad. J. Bot. 42: 213-282.

Maas Geesteranus, R. A. 1963. Hyphal structures in Hydnums II. Proc. Ned. Akad. Wet. (C.) 66: 426-457.

Malençon, G. 1913. La série des Astérosporées. Recueil de travaux crypt. dédiés à L. Mangin 377-396.

Moser, M. 1955. Die Röhrlinge, Blätter- und Bauchpilze, 2. Auf., Kleine Kryptogamenflora IIb, Stuttgart, $327 \mathrm{~S}$.

Overholts, L. O. 1953. The Polyporaceae of the United States, Alaska and Canada. Univ. Michigan Stud., Sci. ser. 19, 466 S., 132 Pl.

Reijnders, A. F. M. 1963. Les problèmes du développement des carpophores des Agaricales et de quelques groupes voisins. Den Haag, $412 \mathrm{~S}$., $55 \mathrm{Pl}$.

Romagnesi, H. 1953. A propos de l'hydne cure-oreille (Auriscalpium vulgare). Bull. Soc. Nat. Oyonnax 7: 111-112.

Singer, R. 1962. The Agaricales in modern taxonomy, 2. ed., Weinheim, 915 S., $73 \mathrm{Pl}$.

und A. H. Smith, 1960. The Asterogastraceous Series. Studies on Secotiaceous Fungi 9. Mem. Torrey Bot. Club 21, Nr. 3, 1-112. 